\title{
Heart failure with reduced ejection fraction: Should we submit patients without angina to coronary angiography?
}

\author{
Filipa Silva a, ${ }^{\mathrm{a}, 1}$, Tiago Borges $^{\mathrm{a}, 1}$, Ana Ribeiro $^{\mathrm{a}}$, Raquel Mesquita ${ }^{\mathrm{a}}$, Olga Laszczynska ${ }^{\mathrm{b}}$, Domingos Magalhães ${ }^{\mathrm{a}}$, \\ João Carlos Silva ${ }^{\mathrm{a}}$, Ana Azevedo ${ }^{\mathrm{b}, \mathrm{c}}$, Paulo Bettencourt ${ }^{\mathrm{a}}$ \\ a Serviço de Medicina Interna, Centro Hospitalar de São João, Porto, Portugal, Serviço de Cardiologia, Centro Hospitalar de São João, Porto, Portugal, \\ UniC - Unidade de investigação e Desenvolvimento Cardiovascular, Porto, Portugal \\ ${ }^{\mathrm{b}}$ EPIUnit - Institute of Public Health of the University of Porto (ISPUP), Porto, Portugal

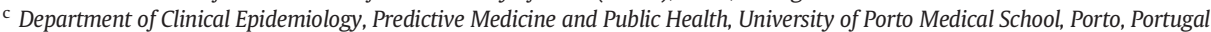

\section{A R T I C L E I N F O}

\section{Article history:}

Received 11 April 2015

Accepted 14 April 2015

Available online 15 April 2015

\section{Keywords:}

Coronary artery disease

Heart failure

Systolic dysfunction

Angiography

Diagnosis

Coronary artery disease (CAD) accounts for about two-thirds of cases of heart failure with reduced ejection fraction (HFrEF) [1]. Angina symptoms and cardiovascular risk factors (CVRF) are not sufficient to establish an ischemic etiology and according to recommendations coronary angiography (CA) is the gold standard [1-3]. There is a paucity of information on the performance of CA for detecting CAD in HFrEF without known CAD [4]. We aimed to determine the frequency of CAD in HFrEF patients of unknown etiology and without known CAD, and to identify variables associated with the presence and severity of CAD.

We retrospectively identified patients from a HF clinic, submitted to CA until the end of 2013. Exclusion criteria were HF with left ventricular ejection fraction $>50 \%$, other etiologies for HF, angina, known CAD (previous myocardial infarction (MI), coronary revascularization) and indication for CA for other purposes. The study protocol conforms to the ethical guidelines of the declaration of Helsinki and was approved by the local ethics committee. Data were abstracted from the clinical records, including demographic variables, HF characteristics, routine laboratory values, CVRF: history of smoking, body mass index, hypertension, dyslipidemia, diabetes mellitus (DM) and family history of premature MI; and comorbidities: atrial fibrillation and chronic kidney

\footnotetext{
* Corresponding author at: Serviço de Medicina Interna, Centro Hospitalar de São João, E.P.E.. Alameda Prof. Hernâni Monteiro, 4200-319 Porto, Portugal.

E-mail address: filipadcs@gmail.com (F. Silva).

1 The first two authors contributed equally to this work.
}

disease. Dyslipidemia, hypertension and DM were defined as either a known previous diagnosis or current prescription for these diseases. The angiographic data included the number of arteries with significant disease and the degree of stenosis. Significant CAD was defined as $\geq 70 \%$ luminal diameter narrowing in at least one coronary artery ( $>50 \%$ for the proximal left anterior descending artery). Severe CAD was defined as the presence of significant CAD of proximal left anterior descending artery, three-vessel CAD or two-vessel CAD involving the left anterior descending artery [5].

Stata version 11.1 for Windows (StataCorp LP, College Station, TX) was used for statistical analysis. Continuous variables are expressed as mean \pm standard deviation, except those without normal distribution, expressed as median (interquartile range). The qualitative variables are expressed as absolute frequencies and percentages. Comparison of above-mentioned variables in both groups (with or without CAD) was performed by using t-test and the Mann-Whitney test for continuous variables with skewed distribution, and the chi-square test for categorical variables. For all analyses, $\mathrm{p}<0.05$ was considered statistically significant.

We included 168 patients with HFrEF who fulfilled the inclusion and exclusion criteria. Baseline characteristics are summarized in Table 1. Of these, 70\% had no CAD, 31\% had significant CAD, among whom $23.1 \%$ had severe CAD. Patients with CAD were older, more frequently male and had a higher prevalence (despite non-significantly) of several CVRF.

The diagnostic yield of CA, in women and men, according to the history of risk factors is summarized in Table 2. Significant CAD was more than twice as prevalent in the presence than in the absence of risk factors. The presence of significant CAD in the latter group was $14.3 \%$ in men and $12.5 \%$ in women.

Our results show that CAD is the etiology of HFrEF in a third of patients without angina and no previous coronary event. Even in the absence of CVRF, more than $10 \%$ of both men and women had significant CAD. These results, although involving a young population, support the routine performance of $\mathrm{CA}$ in $\mathrm{HFrEF}$ independently of CAD risk factors. CAD is the most frequent HFrEF etiology, although the prevalence varies markedly according to populations $[1,6]$. The determination of ischemic etiology in HF patients is of major significance since it can dictate treatment options and ischemic HF is independently associated with a worse long-term outcome $[7,8]$. In patients with angina or MI, 
Table 1

Patient characteristics.

\begin{tabular}{|c|c|c|c|c|c|c|c|}
\hline & \multirow[b]{2}{*}{ All } & \multicolumn{3}{|l|}{ Significant CAD } & \multicolumn{3}{|l|}{ Severe CAD } \\
\hline & & No & Yes & p-Value & No & Yes & p-Value \\
\hline No. of patients & 168 & 112 & 56 & & 129 & 39 & \\
\hline Age (years), mean (SD) & $59.7(11.8)$ & $57.7(11.6)$ & $63.7(11.3)$ & 0.002 & $58.3(11.5)$ & $64.5(11.8)$ & 0.004 \\
\hline Male sex, n (\%) & $119(70.8)$ & $75(67.0)$ & $44(78.6)$ & 0.119 & $89(69.0)$ & $30(76.9)$ & 0.340 \\
\hline LVEF (\%), mean (SD) & $27.0(9.8)$ & $26.1(10.3)$ & $28.5(8.8)$ & 0.232 & $26.6(10.0)$ & $28.2(9.4)$ & 0.483 \\
\hline Severe LVSD, n (\%) & $126(77.3)$ & $84(78.5)$ & $42(75.0)$ & 0.612 & $98(79.0)$ & $28(71.8)$ & 0.347 \\
\hline Diabetes mellitus, n (\%) & $61(36.3)$ & $35(31.3)$ & $26(46.4)$ & 0.054 & $44(34.1)$ & $17(43.6)$ & 0.281 \\
\hline Hypertension, $\mathrm{n}(\%)$ & $98(58.3)$ & $63(56.3)$ & $35(62.5)$ & 0.439 & $75(58.1)$ & $23(59.0)$ & 0.926 \\
\hline Dyslipidemia, n (\%) & $90(53.6)$ & $53(47.3)$ & $37(66.1)$ & 0.022 & $66(51.2)$ & $24(61.5)$ & 0.255 \\
\hline Smoking, n (\%) & $84(53.8)$ & $53(51.5)$ & $31(58.5)$ & 0.404 & $63(52.5)$ & $21(58.3)$ & 0.538 \\
\hline Creatinine $(\mathrm{mg} / \mathrm{dL})$, median (IQR) & $1.03(0.64-2.70)$ & $1.00(0.68-1.79)$ & $1.08(0.67-2.39)$ & 0.352 & $1.01(0.66-2.05)$ & $1.05(0.73-1.48)$ & 0.570 \\
\hline
\end{tabular}

CAD, coronary artery disease; IQR, interquartile range; LVEF, left ventricular ejection fraction; LVSD, left ventricular systolic dysfunction; SD, standard deviation.

Table 2

Diagnostic yield, according to cardiovascular risk factors.

\begin{tabular}{|c|c|c|c|c|c|}
\hline & & \multicolumn{2}{|c|}{ Significant CAD } & \multicolumn{2}{|c|}{ Severe CAD } \\
\hline & & No & Yes & No & Yes \\
\hline \multirow[t]{2}{*}{ Women } & No risk factors, $\mathrm{n}(\%)^{\mathrm{a}}$ & $14(87.5)$ & $2(12.5)$ & $14(87.5)$ & $2(12.5)$ \\
\hline & Any risk factor, $\mathrm{n}(\%)^{\mathrm{a}}$ & $23(69.7)$ & $10(30.3)$ & $26(78.8)$ & $7(21.2)$ \\
\hline \multirow[t]{2}{*}{ Men } & No risk factors, $\mathrm{n}(\%)^{\mathrm{a}}$ & $12(85.7)$ & $2(14.3)$ & $12(85.7)$ & $2(14.3)$ \\
\hline & Any risk factor, $\mathrm{n}(\%)^{\mathrm{a}}$ & $63(60.0)$ & $42(40.0)$ & $77(73.3)$ & $28(26.7)$ \\
\hline
\end{tabular}

$\mathrm{CAD}$, coronary artery disease.

a Risk factors: diabetes mellitus, dyslipidemia, and smoking.

the extent of CAD, defined by angiography, is a predictor of mortality. Also, the extent of CAD is a significant risk factor for cardiovascular outcomes after MI is complicated by HF [9]. Additionally, patients with ischemic HF may benefit from secondary preventive measures and coronary revascularization $[1,3,4]$.

According to the 2014 guidelines on myocardial revascularization, CA continues to be the gold standard for the assessment of CAD severity $[2,3,9]$. Some authors propose routine angiography in all patients with HFrEF, because the presence of CAD is not uncommon among patients with HF of unknown etiology [10]. In our population of 168 patients with HFrEF and no angina, the prevalence of angiographically significant CAD was high (31\%).

A clinical tool has been recently validated to help clinicians predict the absence of severe CAD in patients with HFrEF and therefore eliminate unnecessary CA. Independent predictors of severe CAD included $\mathrm{DM}$, electrocardiographic $\mathrm{Q}$ waves or left bundle branch block and at least two nondiabetic risk factors. The presence of at least one of these factors identified more than 95\% of CAD cases and all of the patients with severe disease [10]. This study, as ours, was limited, by having included only those patients who had undergone angiography.

Our results corroborate previous observations, showing similar rates of CAD in HFrEF patients without angina $[4,10]$. In our study, although patients with significant CAD had a higher prevalence of CVRF they were not statistically significantly different from patients without CAD. We observed that both male groups (with or without CVRF) had significant CAD, which support the use of CA. Even $12.1 \%$ of women without CVRF had significant CAD.

Our study is a retrospective analysis with limitations and biases that are inherent. Besides being a single center study, the patients were submitted to CA at the physicians' discretion. Also, known CAD was based on the reported symptoms of typical angina and a history of previous MI or coronary revascularization, which may have been underreported. The relatively small sample size limits the generalization of results and can underestimate the interactions between CVRF and CAD. Additionally our population was relatively young, precluding generalization of the observations, but one can speculate that with increasing age the yield would be even higher, as CAD prevalence increases with age.

In conclusion, our study shows that among HFrEF patients without angina the prevalence of angiographically significant CAD was high, suggesting that the performance of routine CA should be considered in HFrEF patients.

\section{Conflict of interest}

The authors report no relationships that could be construed as a conflict of interest.

\section{References}

[1] J. McMurray, S. Adamopoulos, S. Anker, et al., ESC guidelines for the diagnosis and treatment of acute and chronic heart failure 2012, Eur. Heart J. 33 (14) (2012) 1787-1847.

[2] S. Windecker, P. Kolh, F. Alfonso, et al., 2014 ESC/EACTS guidelines on myocardial revascularization, Eur. J. Cardiothorac. Surg. 46 (2014) 517-592.

[3] L.D. Hillis, P.K. Smith, J.L. Anderson, et al., 2011 ACCF/AHA guideline for coronary artery bypass graft surgery: executive summary: a report of the American College of Cardiology Foundation/American Heart Association Task Force on practice guidelines, Circulation 124 (2011) 2610-2642.

[4] W. Pitts, R. Lange, D. Hillis, J. Cigarroa, Coronary arterial anatomy in patients with left ventricular systolic dysfunction without chest pain or previous myocardial infarction, Am. J. Cardiol. 82 (1998) 1530-1531.

[5] G. Levine, E. Bates, J. Blankenship, et al., 2011 ACCF/AHA/SCAI guideline for percutaneous coronary intervention, J. Am. Coll. Cardiol. 58 (2011) e44-e122.

[6] M. Gheorghiade, G. Sopko, L. De Luca, et al., Navigating the crossroads of coronary artery disease and heart failure, Circulation 114 (2006) 1202-1213.

[7] J. McMurray, Systolic heart failure, N. Engl. J. Med. 362 (2010) 228-238.

[8] K.F. Adams Jr., S.H. Dunlap, C.A. Sueta, et al., Relation between gender, etiology and survival in patients with symptomatic heart failure, J. Am. Coll. Cardiol. 28 (1996) 1781-1788.

[9] R. Janardhanan, S. Kenchaiah, E. Velasquez, et al., Extent of coronary artery disease as a predictor of outcomes in acute myocardial infarction complicated by heart failure, left ventricular dysfunction, or both, Am. Heart 152 (2006) 183-189.

[10] R. Doukky, M.J. Shih, M. Rahaby, et al., A simple validated clinical tool to predict the absence of coronary artery disease in patients with systolic heart failure of unclear etiology, Am. J. Cardiol. 112 (2013) 1165-1170. 